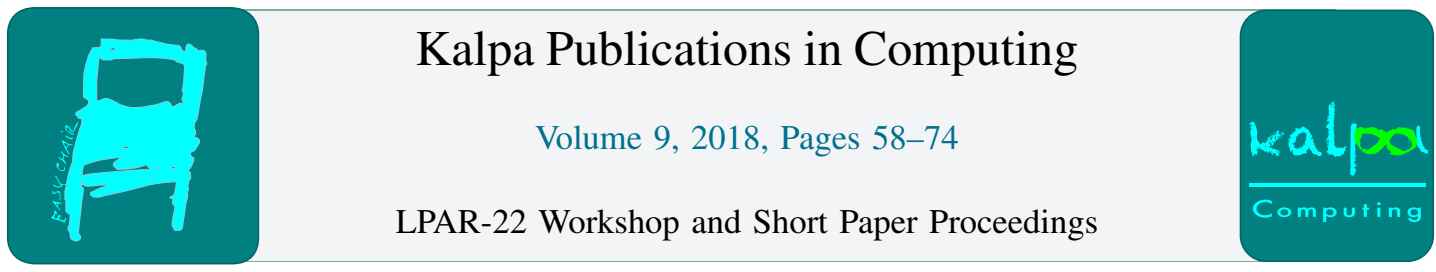

\title{
Symmetry breaking in a new stable model search method
}

\author{
Tarek Khaled and Belaid Benhamou \\ Aix Marseille University, University of Toulon, CNRS, LIS, Marseille, France. \\ \{tarek.khaled, belaid.benhamou\}@univ-amu.fr
}

\begin{abstract}
In this work, we investigate the inclusion of symmetry breaking in the answer set programming (ASP) framework. The notion of symmetry is widely studied in various domains. Particularly, in the field of constraint programming, where symmetry breaking made a significant improvement in the performances of many constraint solvers. Usually, combinatorial problems contain a lot of symmetries that could render their resolution difficult for the solvers that do not consider them. Indeed, these symmetries guide the solvers in the useless exploration of symmetric and redundant branches of the search tree. The ASP framework is well-known in knowledge representation and reasoning. However, only few works on symmetry in ASP exist. We propose in this paper a new ASP solver based on a novel semantics that we enhance by symmetry breaking. This method with symmetry elimination is implemented and used for the resolution of a large variety of combinatorial problems. The obtained results are very promising and showcase an advantage when using our method in comparison to other known ASP methods.
\end{abstract}

\section{Introduction}

Answer Set Programming (ASP) is an important framework that is used to express and solve a variety of high combinatorial problems, such as graph problems, planning and model checking. ASP is applied in robotics, computational biology and also for industrial purposes. It is an expressive modeling tool, that is able to encode an important number of problems. It has become a popular approach because of the availability of several efficient software tools like Clasp [14], DLV [18], Smodels [25] and other systems based on SAT solver like ASSAT [20] and Cmodels [19]. The ASP paradigm emerged from the research on knowledge representation and non-monotonic reasoning. Several works have been done to define semantics for logic programs. The main objective behind them is to give a precise meaning to the negation as failure (default negation). The first semantics had been proposed by Clark [8]. This semantics is used in several ASP solvers, but the most known semantics in ASP is the one of stable models [16]. Lately, a new semantics has been proposed [7]. In this semantics, a logic programs is represented by a set of Horn clauses that has the same size as the input ground program. The benefit of this semantics is the easy characterization of the stable models and the extension it provides to the stable model semantics.

Here after, we propose a new method for answer set searching that rely on a Boolean enumeration process defined for the ASP paradigm according to the semantics introduced in [7]. This method has the advantage of performing the enumerative process only on a restricted set of literals called the strong backdoor (STB) [26] of the logic program. The search method computes all the possible extensions of 
the source logic program from which we can generate all the stable models. It could also compute extramodels corresponding to a kind of extra-extensions that are not captured by the stable model semantics. These extra-models extend the stable model semantics. The stable models are deduced from a sub-set of extensions satisfying what is called here a a discriminant condition.

On the other hand, symmetry is studied in several fields including mathematics and artificial intelligence. An object is symmetrical, when the permutation of its elements leaves the object unchanged. Symmetry is a fundamental notion in the satisfiability problem that permit to reduce the computational complexity when dealing with combinatorial problems. The principle of symmetry in propositional logic has been first introduced by Krishnamurthy in [17]. Symmetry has been studied in depth in [4, 5] where a dynamic symmetry elimination is proposed for the DPLL method [11] when solving the satisfiability problem. A static approach that breaks symmetries in a preprocessing phase is introduced in [9]. This static approach consists in adding constraints expressing the global symmetry of the initial representation of the problem. This technique has been improved in [2]. Several combinatorial problems when expressed in the ASP paradigm hold a great number of symmetries. For instance the Pigeon Hole problem is known to require exponential time to be solved when the symmetries are not eliminated. Indeed, the ASP solver explores all the symmetrical search spaces. It is possible to avoid exploring these redundant search spaces by breaking the existing symmetries. Until now, only few works on symmetry elimination in ASP have been done. For instance the method presented in [12] treats symmetry in ASP with a statical approach and the encoding is based on the body-atom representation. A different approach is studied in [6]. This last method breaks symmetry in both a statical and a dynamical way.

In this paper, we first start by discussing the new method for ASP solving, then deal with the detection and the elimination of the symmetries of the Horn clausal representation that the new semantics [7] uses to express logic programs. We split the problem of symmetry breaking into three parts. We start by creating a colored graph that represents the Horn clausal form of the given logic program in such a way that the automorphisms of the graph are identical to the symmetries of the Horn clausal representation. Then, a set of generators representing the automorphism group of the graph is computed by using tools like saucy [2], autom [24] or nauty [21]. Finally, symmetry-breaking predicates (SBP) are constructed and added to the Horn clausal formulation. Our approach is inspired by the works presented in $[2,3]$.

The rest of the paper is organized as follows. First, we recall in Section 2 some notions on logic programming and the semantics [7] on which our search method is based. Then we describe in Section 3 the new ASP search method. In Section 4, we give the definition and the theoretical properties of symmetry. We describe in Section 5 the symmetry detection method before discussing the symmetrybreaking approach in Section 6. Section 7 gives the experimental results obtained on some combinatorial problems. Section 8 concludes the work.

\section{Background}

We will summarize in the following the notions of permutations, the answer set programming framework and the main theoretical bases of the used semantics [7].

\subsection{Permutations}

Let $\Omega=\{1,2, \ldots, N\}$ for some integer $N$, where each integer might represent a propositional variable or an atom. A permutation of $\Omega$ is a bijection mapping defined from $\Omega$ into itself. We denote by $\operatorname{Perm}(\Omega)$ the set of all permutations of $\Omega$. The pair $(\operatorname{Perm}(\Omega), \circ)$ where $\circ$ is the composition of the permutations of $\operatorname{Perm}(\Omega)$ forms the permutation group of $\Omega$. The orbit of an element $\omega$ on which the group $\operatorname{Perm}(\Omega)$ acts is $\omega^{\operatorname{Perm}(\Omega)}=\left\{\omega^{\sigma} \mid \omega^{\sigma}=\sigma(\omega), \sigma \in \operatorname{Perm}(\Omega)\right\}$. In other words, the orbit of an element $\omega$ under a permutation group are the set of all elements to which the element $\omega$ can be mapped. 
A generating set of the group $\operatorname{Perm}(\Omega)$ is a subset Gen of $\operatorname{Perm}(\Omega)$ such that each element of $\operatorname{Perm}(\Omega)$ can be written as a composition of elements of Gen. We write $\operatorname{Perm}(\Omega)=\langle$ Gen $\rangle$. An element of Gen is called a generator. The orbit of $\omega \in \Omega$ can be computed by using only the set of generators Gen.

\subsection{Answer Set Programming}

A logic program $\pi$ is a finite set of rules of the form $r$ :head $(r) \leftarrow \operatorname{body}(r)$, where $\operatorname{body}(r)$ is the set of premises of the rule given as conjunction of literals. The head(r) represent the conclusion of the rule which is generally a single literal or in some case a disjunction of literals for disjunctive logic programs. It is in general, given in first order logic and grounders like gringo [15] or lparse [23] are used to compute all its ground instances. In the sequel, we focus on ground programs, we assume that $\pi$ is ground.

There are different classes of logic programs. They differ by the presence or the absence of the classical negation and the negation as failure in the rules of the program. A positive logic program $\pi$ is a set of rules of the form : $r=A_{0} \leftarrow A_{1}, A_{2}, \ldots, A_{m}$, with $(m \geq 0)$ and where $A_{i}$ is an atom for $0 \leq i \leq m$. There is no classical negation or negation as failure in a positive logic program. A general logic program $\pi$ is a set of rules of the form : $r=A_{0} \leftarrow A_{1}, A_{2}, \ldots, A_{m}$, not $A_{m+1}, \ldots$, not $A_{n},(0 \leq m<n)$ where $A_{i}$ is an atom for $0 \leq i \leq n$ and not the symbol expressing the negation as failure. The positive body of $r$ is $b_{0 d y^{+}}(r)=\left\{A_{1}, A_{2}, \ldots, A_{m}\right\}$ and the negative is $b_{0 d y}(r)=\left\{A_{m+1}, \ldots, A_{n}\right\}$. The positive projection of $r$ is $r^{+}=A_{0} \leftarrow A_{1}, A_{2}, \ldots, A_{m}$. The intuitive meaning of the rule $r$ is the following: if we prove all the atoms of $\operatorname{body}^{+}(r)$ and at the same time no atom of $\operatorname{body}^{-}(r)$ had been proven, then we infer the head $A_{0}$. The reduct of a program $\pi$ with respect to a given set of atoms $X$ is the positive program $\pi^{X}$ obtained from $\pi$ by deleting each rule containing a default-negated atom not $A_{i}$ in its negative body such that $A_{i} \in X$ and all the atoms not $A_{j}$ from the remaining rules. Formally, $\pi^{X}=\left\{r^{+}:\right.$body $\left.y^{-}(r) \cap X=\emptyset\right\}$. Throughout the rest of the paper, we will focus on general logic programs.

The most known semantics for logic programs is the one of stable models [16]. A set $X$ of atoms is a stable model of $\pi$ iff $X$ is identical to the minimal Herbrand model of the reduct $\pi^{X}$ obtained from $\pi$ when considering the set of atoms $X$. This model is also called the canonical model of $\pi^{X}$, it is denoted by $\operatorname{Cn}\left(\pi^{X}\right)$. That is, a set $X$ of atoms is a stable model of $\pi$ if and only if $X=\operatorname{Cn}\left(\pi^{X}\right)$. Clark completion semantics [8] is also used to compute the stable models. It is known that each stable model of logic program $\pi$ is a model of its completion, but a model of the completion is not always a stable model [13] of $\pi$. Loop formulas are added to the completion to establish the equivalence between the stable models of $\pi$ and the models of its Clark completion [20]. The size of the CNF formula resulting from the loop management could vary exponentially within the size of the program. The ASP solvers that use this approach could have an exponential space complexity in the worst case.

\subsection{The used semantics}

A new semantics is proposed in [7]. This semantics uses a Horn clausal representation to express the considered logic program. This representation has the advantage of having the same size as the one of the input logic program. The new semantics is based on a classical propositional language $L$ composed by two types of literals, a subset of classical literals $V=\left\{A_{i}: A_{i} \in L\right\}$ and an other subset $n V=\left\{\right.$ not $A_{i}:$ not $\left.A_{i} \in L\right\}$. For each literal $A_{i} \in V$, there is a corresponding literal not $A_{i} \in n V$ designing the negation as failure of $L_{i}$. A connection between these two types of literals is expressed by the addition to the propositional language $L$ of an axiom expressing the mutual exclusion between each literal $A_{i} \in V$ and its corresponding negative literal not $A_{i} \in n V$. This axiom of mutual exclusion is expressed by a set of clauses $M E=\left\{\left(\neg A_{i} \vee \neg\right.\right.$ not $\left.\left.A_{i}\right): A_{i} \in V\right\}$. A ground general logic program $\pi=\left\{r: A_{0} \leftarrow\right.$ 
$A_{1}, A_{2}, \ldots, A_{m}$, not $A_{m+1}, \ldots$, not $\left.A_{n}\right\},(0 \leq m<n)$ is expressed in the propositional language $L$ by a set of Horn clauses $C R=\left\{\bigcup_{r \in \pi}\left(A_{0} \vee \neg A_{1} \vee, \ldots, \neg A_{m} \vee \neg\right.\right.$ not $A_{m+1}, \ldots, \neg$ not $\left.\left.A_{n}\right)\right\},(0 \leq m<n)$ representing all the rules of the logic program to which the set of mutual exclusion clauses $M E=\left\{\left(\neg A_{i} \vee \neg\right.\right.$ not $\left.\left.A_{i}\right): L_{i} \in V\right\}$ is added. The Horn clausal form of $\pi$ is the following :

$$
\mathrm{HC}(\pi)=\left\{\bigcup_{\mathrm{r} \in \pi}\left(\mathrm{A}_{0} \vee \neg \mathrm{A}_{1} \vee, \ldots, \neg \mathrm{A}_{\mathrm{m}} \vee \neg \operatorname{not} \mathrm{A}_{\mathrm{m}+1}, \ldots, \neg \text { not } \mathrm{A}_{\mathrm{n}}\right) \underset{\mathrm{A}_{\mathrm{i}} \in \mathrm{V}}{\bigcup}\left(\neg \mathrm{A}_{\mathrm{i}} \vee \neg \text { not } \mathrm{A}_{\mathrm{i}}\right)\right\} .
$$

The size of the complete representation of the logic program $\pi$ is approximately equal to size $(\pi)+$ $2 n$, where $n=|V|$. The factor $2 n$ corresponds to the set of clauses $M E$.

The algorithm that we will present in the next section operates on the form $H C(\pi)$. It is an enumerative algorithm that performs on a subset of variables that represent the strong backdoor (STB)[26] of the input logic program. The set STB is formed by the literals of the form not $A_{i}$ that occur in the logic $\operatorname{program} \pi$.

Definition 1. Given a logic program $\pi$, its strong backdoor is $S T B=\left\{\right.$ not $A_{i} \in n V: \exists r \in \pi$, not $A_{i} \in$ body $\left.y^{-}(r)\right\}$.

The method mainly computes the extensions of $H C(\pi)$ that encodes the stable models. Given a program $\pi$ and its STB. An extension of $H C(\pi)$ with respect to the STB (or simply an extension of the pair $(H C(\pi), S T B)$ is the set of consistent clauses derived from $H C(\pi)$ when adding a maximal set of literals not $A_{i} \in S T B$. In other word, an extension of $\left.(H C(\pi), S T B)\right)$ is maximally consistent with respect to inclusion of literals not $A_{i} \in S T B$, when the addition of one more literal not $A_{i} \in S T B$ renders the resulting extension inconsistent. Formally:

Definition 2. Let $H C(\pi)$ be the Horn CNF encoding of a logic program $\pi$, STB its strong backdoor and $S \subseteq S T B$. The set $\mathrm{E}=\mathrm{HC}(\pi) \cup \mathrm{S}$ of clauses is then an extension of $(\mathrm{HC}(\pi), \mathrm{STB})$ if the following conditions hold:

1. E is consistent,

2. $\forall \operatorname{not} \mathrm{A}_{\mathrm{i}} \in \mathrm{STB}-\mathrm{S}, \mathrm{E} \cup\left\{\operatorname{not} \mathrm{A}_{\mathrm{i}}\right\}$ is inconsistent.

It is shown in [7], that each stable model of a $\operatorname{logic}$ program $\pi$ corresponds to an extension $E$ of $H C(\pi)$ that satisfies the discriminant condition $\left(\forall A_{i} \in V, E=\neg\right.$ not $\left.A_{i} \Rightarrow E \mid=A_{i}\right)$ and vice-versa. There is a one to one bijection between the stable models of $\pi$ and these extensions. The main proved theoretical properties are the following:

Theorem 1. If $E$ is an extension of $(H C(\pi), S T B)$, that verify the discriminant condition: $\forall A_{i} \in V, E \models$ $\neg$ not $A_{i} \Rightarrow E=A_{i}$, then $\mathrm{X}=\left\{\mathrm{A}_{\mathrm{i}}: \mathrm{E}=\mathrm{A}_{\mathrm{i}}\right\}$ is a stable model of $\pi$.

It is also shown in [7] that for each stable model of the logic program there exists an extension of $(H C(\pi), S T B)$ from which it could be deduced.

Theorem 2. If $X$ is a stable model of a logic program $\pi$, then there exist an extension $E$ of $(L(\pi), S T B)$ such that $X=\left\{L_{i} \in V: E \mid=L_{i}\right\}$ and which verifies the discriminant condition $\left(\forall L_{i} \in V, E \mid={ }^{1} \neg\right.$ not $L_{i} \Rightarrow$ $\left.E \models L_{i}\right)$.

Example 1. Consider the logic program $\pi=\{a \leftarrow$ not $b ; b \leftarrow$ not $a\}$ The Horn clausal representation of the logic program $\pi$ is formed by the set $H C(\pi)=C R \cup M E$ where $C R=\{a \vee \neg$ not $b, b \vee \neg$ not $a\}, M E=$ $\{\neg a \vee \neg$ not $a, \neg b \vee \neg$ not $b\}$ and its strong backdoor is $S T B=\{$ not $a$, not $b\}$. We can see that $(H C(\pi), S T B)$ admits two extensions $E_{1}=H C(\pi) \cup\{$ not $a\}$ and $E_{2}=H C(\pi) \cup\{$ not $b\}$. Indeed, $E_{1}$ and $E_{2}$ are maximally consistent with respect to the set STB. The two extensions satisfy the discriminant condition. Thus, the logic program has two stable models $M_{1}=\{b\}$ and $M_{2}=\{a\}$ that are deduced from $E_{1}$ resp. $E_{2}$ by unit resolution.

\footnotetext{
${ }^{1}$ The symbol $\models$ expresses the classical logical infrence
} 


\section{The proposed search method}

We describe here the new search method for stable models that is based on the semantics summarized previously [7]. For a given logic program $\pi$, this method computes all the extensions of $(H C(\pi), S T B)$ from which the stable models are deduced by unit resolution. Intuitively, the search of the extensions of $(H C(\pi), S T B)$ is done by the progressive addition of literals not $A_{i}$ of the $S T B$ to $H C(\pi)$ and checking the consistency of the obtained set at each node. If we focus only on stable models, then we just have to look after the extensions verifying the discriminant condition. In other words, we prune the search tree to remove the extra-extensions which don't verify that condition. The proposed method is able to compute all the stable models of a given logic program and in general could search extra-models when stable models do not exist. However, in this work, we limited the search to only stable models. We did this in order to have a safe comparison with other ASP systems that consider only stable models. The enumeration process builds incrementally an extension by alternating in the search tree between deterministic nodes corresponding to the unit propagations and non deterministic nodes that are the choice points. The choice points are defined by the affectation of truth values (true or false) to some literals of the strong backdoor set $S T B$. Some new inference rules that the method uses to increase the number of unit propagations and then reduces the search space are introduced.

\subsection{The theoretical bases of the method}

We will now introduce some inference rules that the method will use thereafter in the enumeration process.

Definition 3. Let $\pi$ be a logic program and $H C(\pi)$ its Horn clausal representation. We define on $H C(\pi)$ two inference rules: $\frac{A_{i}}{\neg \text { not } A_{i}}$ and $\frac{\text { not } A_{i}}{\neg A_{i}}$.

These two inference rules represent an efficient implementation of the set of clauses

$M E=\bigcup_{A_{i} \in V}\left\{\left(\neg A_{i} \vee \neg\right.\right.$ not $\left.\left.A_{i}\right)\right\}$ of $H C(\pi)$ expressing the mutual exclusion between each pair of atoms $A_{i}$ and $\operatorname{not} A_{i}$.

In the case of our method, the enumeration is done only on the subset of STB literals. Let $C_{S T B}=$ $\left\{c_{i}=\neg\right.$ not $A_{i_{1}} \vee, \ldots, \vee \neg$ not $A_{i_{k}} /\left|c_{i}\right| \geq 1, \forall j \in\{1 . . k\}$, not $\left.A_{i_{j}} \in S T B\right\}$ be the set of all possible negative clauses formed by some literals of the set $S T B$ and which have at least one literal. The non deterministic treatment of a choice point corresponding to a strong backdoor literal not $A_{j}$ is done by first its assignment to the value true to favor the current extension maximality. The exploration of the branch corresponding to the assignment of the truth value false to not $A_{j}$ is necessary only when the first branch produced at least one sub clause $c_{i} \in C_{S T B}$. This property, could considerably reduce the complexity of the studied method, we will prove it in Proposition 1.

Proposition 1. Let $\pi$ be a logic program, $H C(\pi)$ its Horn clausal form, $H C(\pi)_{I}$ its Horn clausal form simplified by the partial interpretation I corresponding to the current node $n$ of the search tree, $S T B=\left\{\right.$ not $A_{i}: \exists r \in \pi$, not $\left.A_{i} \in r\right\}$ the strong backdoor of $\pi$, and $C_{S T B}$ the set of all possible negative clauses formed by literals of the set STB. If not $A_{j} \in S T B$ is the current literal to assign at the node $n$ and the condition $\forall c_{i} \in C_{S T B}, H C(\pi)_{I} \wedge$ not $A_{j} \not \forall c_{i}$ holds, then each extension of $H C(\pi)_{I} \wedge \neg$ not $A_{j}$ is also an extension of $H C(\pi)_{I} \wedge$ not $A_{j}$.

Proof. The subset of clauses $H C(\pi)_{I}$ corresponding to the current node $n$ of the search tree is the simplified clause system obtained from $H C(\pi)$ by the consideration of the literals that are assigned in the partial interpretation I. By the hypothesis, the literal not $A_{j}$ is the next element of STB that will be assigned at the node $n$. The set $H C(\pi)_{I}$ has two types of clauses : the subset of clauses of the form $\neg$ not $A_{j} \vee C_{1}$ containing the literal $\neg$ not $A_{j}$ and 
where $C_{1}$ represent a set of pieces of clauses, and the subset of clauses $C_{2}$ that do not contain the literal $\neg$ not $A_{j}$. Let $e=$ not $A_{i_{1}} \wedge \ldots \wedge$ not $A_{i_{k}}$ be an extension of $H C(\pi)_{I} \wedge \neg$ not $A_{j}$ where not $A_{i_{j}} \in S T B$. We shall prove that $e$ is also an extension of $H C(\pi)_{I} \wedge$ not $A_{j}$. We can see that $H C(\pi)_{I} \wedge \neg$ not $A_{j} \equiv C_{2}$ and $H C(\pi)_{I} \wedge$ not $A_{j} \equiv C_{1} \wedge C_{2}$. The set $e$ is an extension of $H C(\pi)_{I} \wedge \neg$ not $A_{j}$, thus $C_{2} \wedge e$ is consistent. To show that $e$ is also an extension of $H C(\pi)_{I} \wedge$ not $A_{j}$, it is sufficient to prove that $C_{1} \wedge C_{2} \wedge e$ is consistent. We proceed by contradiction. That is, by supposing that $C_{1} \wedge C_{2} \wedge e$ is inconsistent. It results that $C_{1} \wedge C_{2} \wedge e=\square$ and thus $C_{1} \wedge C_{2} \models \neg e$. This means that $C_{1} \wedge C_{2} \models \neg$ not $A_{i_{1}} \vee \ldots \vee \neg$ not $A i_{k} \in C_{S T B}$. Therefore, we have $H C(\pi)_{I} \wedge$ not $A_{j} \models \neg$ not $A_{i_{1}} \vee \ldots \vee \neg$ not Ai $i_{k} \in C_{S T B}$. Thus, $H C(\pi)_{I} \wedge$ not $A_{j}=c_{i} \in C_{S T B}$ and this contradicts the assumption

In other words, if no clause $c_{i} \in C_{S T B}$ had been produced at a choice point of the search tree where the value true is assigned to a literal not $A_{j} \in S T B$, then there is no need to explore the branch corresponding to the negative literal $\neg n o t A_{j}$. This could avoid to the method to explore redundant and pointless branches.

Proposition 2. If $\mathrm{HC}(\pi)$ is the clausal representation of a logical program $\pi$ and I the current partial interpretation, then the unit resolution is sufficient to produce from $H C(\pi)_{I}$ any clause $c_{i}=\neg$ not $A_{i_{1}} \vee$ $\ldots \vee \neg n o t A i_{k} \in C_{S T B}$.

Proof. The automatic deduction theorem states that proving $H C(\pi)_{I}=c_{i}$ is equivalent to $H C(\pi)_{I} \wedge \neg c_{i} \models \perp$. Since $H C(\pi)$ is a set of Horn clauses, it follows that the simplified set of clauses $H C(\pi)_{I} \wedge \neg c_{i}$ is also of Horn. That is, $H C(\pi)_{I} \wedge$ not $A i_{1} \wedge \ldots \wedge n o t A i_{k}$ is a set of Horn clauses. Since the unit resolution is sufficient to decide the consistency of any set of Horn clauses, then it is in particularly true for $H C(\pi)_{I} \wedge \neg c_{i}$. In other words, the unit resolution is sufficient to show $H C(\pi)_{I} \wedge \neg c_{i} \models \perp$ and therefore sufficient to show $H C(\pi)_{I} \models c_{i}$

To apply the cut induced by Proposition 1 at a given choice point of the search tree, our method must prove that no sub-clause $c_{i} \in C_{S T B}$ is produced at that node. To do this, the method tries to produce such a clause by unit resolution (Proposition 2).

Now, we will show how to exploit the apparition of negative pure (monotone) literals during the search. These literals are frequently overlooked in the implementation of SAT solvers based on DPLL, but for the ASP solvers, they perform a critical role.

Proposition 3. Let $\pi$ be a logic program, $H C(\pi)$ its Horn clausal representation and $\neg A_{i}$ a pure literal of $H C(\pi)$, if $X$ is a stable model of $\pi$ then $\neg A_{i} \in X$.

Proof. The literal $\neg A_{i}$ is pure in $H C(\pi)$. This means that $A_{i}$ has no occurrence in $H C(\pi)$, thus the literal $A_{i}$ can never be inferred. Therefore, $A_{i}$ can never be a part of a stable model $X$. By applying the closed world assumption, we have $\neg A_{i} \in X$

This proposition allows to propagate pure negative literals such as mono-literals. Such propagations contribute to the reduction of the number of choice points in the search tree.

Proposition 4. Let $\pi$ be a logic program and $H C(\pi)$ its clausal representation, if $\neg A_{i}$ is true in a stable model $X$ of $\pi$ then not $A_{i}$ must be true in $X$.

Proof. If $\neg A_{i}$ is true in the stable model $X$, then the only case where $\neg$ not $A_{i}$ can be produced is the existence of a sub-clause $A_{i} \vee \neg n o t A_{i}$ of $H C(\pi)_{X}$. But in this case, the corresponding extension to $X$ does not verify the discriminant condition. Hence, $X$ would not be a stable model, and this contradicts the assumption

The previous proposition defines an inference rule $\left(\frac{\neg A_{i}}{\operatorname{not} A_{i}}\right)$ that the method will use to prune the search tree.

Proposition 5. Let $\pi$ be a logic program and $H C(\pi)$ its corresponding clausal form, if $\neg$ not $A_{i}$ is true in a partial model $X$ of $\pi$ and $A_{i}$ is false, then $X$ can not be extended to stable model.

Proof. If $\neg$ not $A_{i}$ is true in the partial model $X$ and at the same time $A_{i}$ is false in it, then the corresponding extension to $X$ does not verify the discriminant condition. Hence, $X$ can not be extended to a stable model of $\pi \quad \square$

All the propositions mentioned above bring cuts in the search tree and reduce considerably the search space. 


\subsection{The algorithm description}

In the following, we present the new search algorithm for stable models. Its enumerative process explores a boolean tree search. It looks like the one of a DPLL [11] procedures that is adapted to the ASP framework and to the used semantics [7]. We implemented all the inferences rules introduced previously, to boost the method. The main search process alternates between deterministic unit propagation phases and non deterministic choice point phases where STB clause production is launched on the first branch where a literal not $A_{i}$ of the STB is interpreted to the value true. That is, if no clause $\left(c_{i} \in C_{S T B}\right)$ is produced, then the branch assigning the value false to $\operatorname{not} A_{i}$ is not explored. The production process is useless on the last branch. Throughout the two alternate phases, the algorithm affect truth values to literals and develops a similar tree search as the one of a DPLL procedure. If a conflict is encountered during the search, then the algorithm explores the second branch corresponding to the second truth value of the literal representing the current choice point only if a clause $c_{i} \in C_{S T B}$ is produced. otherwise a backtrack is done. An extension candidate is founded either when all the clauses are satisfied, or when all the literals of STB are affected without falsifying any clause. In both cases, the algorithm execute a completing phase that consists in completing the current interpretation by assigning the value true to all the remaining literals not $A_{i}$ of $n V$ and by assigning the value false to all the others literals $A_{i} \in V$ not assigned yet according to the closed world assumption.

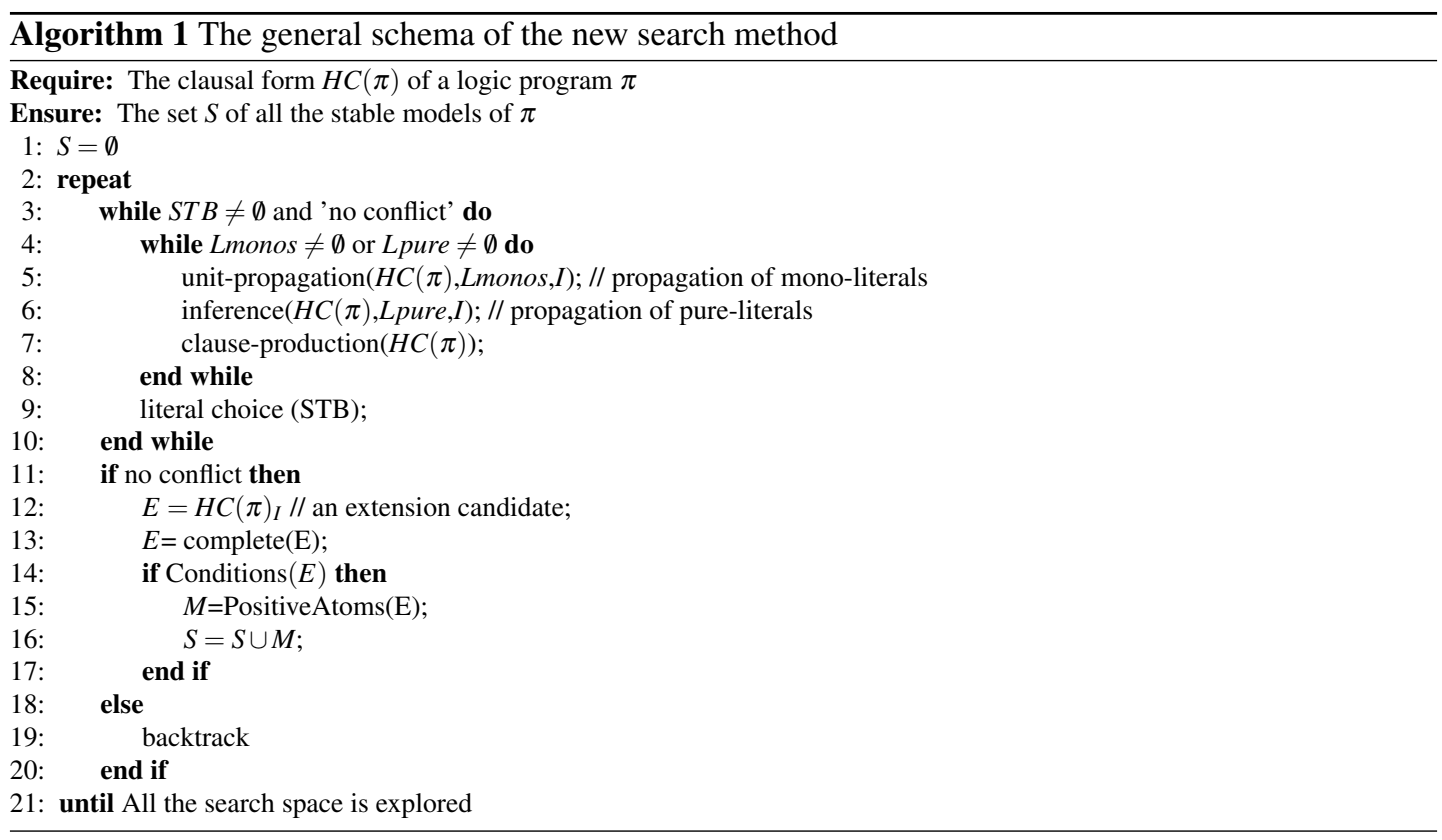

The algorithm starts by a first call to the unit-propagation procedure to propagate all the monoliterals ${ }^{2}$ until the list of mono-literals Lmono becomes empty. Then it deals with the pure literals which also could induce mono-literals. When there is no mono-literal and no pure literals to assign, the algorithm tries to produce a clause $c_{i} \in C_{S T B}$ (proposition 2). If we produce a clause $c_{i} \in C_{S T B}$, then the second branch of the current choice point will be explored. Otherwise, if no clause was produced and all the mono-literals and the pure literals are treated, then the second branch of the choice point literal become useless. The enumeration continue by choosing in STB the next literal to assign. This process is repeated either until the satisfaction of all the clauses, or until the assignation of all the literals of

\footnotetext{
${ }^{2} \mathrm{~A}$ mono-literal means a unit clause
} 
$S T B$ without the appearance of the empty clause. An extension candidate $E=H C(\pi)_{I}$ is obtained when we reach this state and the completing phase is performed to get a kind of minimal model. After the verification of the maximality and the discriminant conditions on $E$, a stable model $M$ consisting of the positive atoms $A_{i}$ of $E$ is extracted and added to the set $S$. The pseudo-code of the general schema of the method is given in Algorithm 1.

The unit-propagation procedure (Algorithm 2) takes as inputs the clausal form $H C(\pi)$, the list of unit clauses Lmonos, and the current partial interpretation $I$. It returns either an extended interpretation of $I$ or a conflict message if an empty clause is found. The procedure starts by satisfying all the clauses where a certain mono-literal $v$ appears and add $v$ to the partial interpretation $I$. Then, it reduces the clauses where the opposite of $v$ (the literal $\neg v$ ) appears. If a unit clause is produced, it will be added to the list of mono-literals Lmonos. If an empty clause is detected, the procedure reports the conflict. Secondly, the algorithm calls the Inference function that implements the inference rules seen in the previous subsection. Such inference rules lead to reduce the set of choice points in the search tree. That is, the literal $v^{\prime}$ returned by the inference procedure will be processed like a mono-literal. Finally, the method calls the clauses-production procedure that uses unit resolution to produce the clauses $c_{i} \in C_{S T B}$ according to Proposition 2.

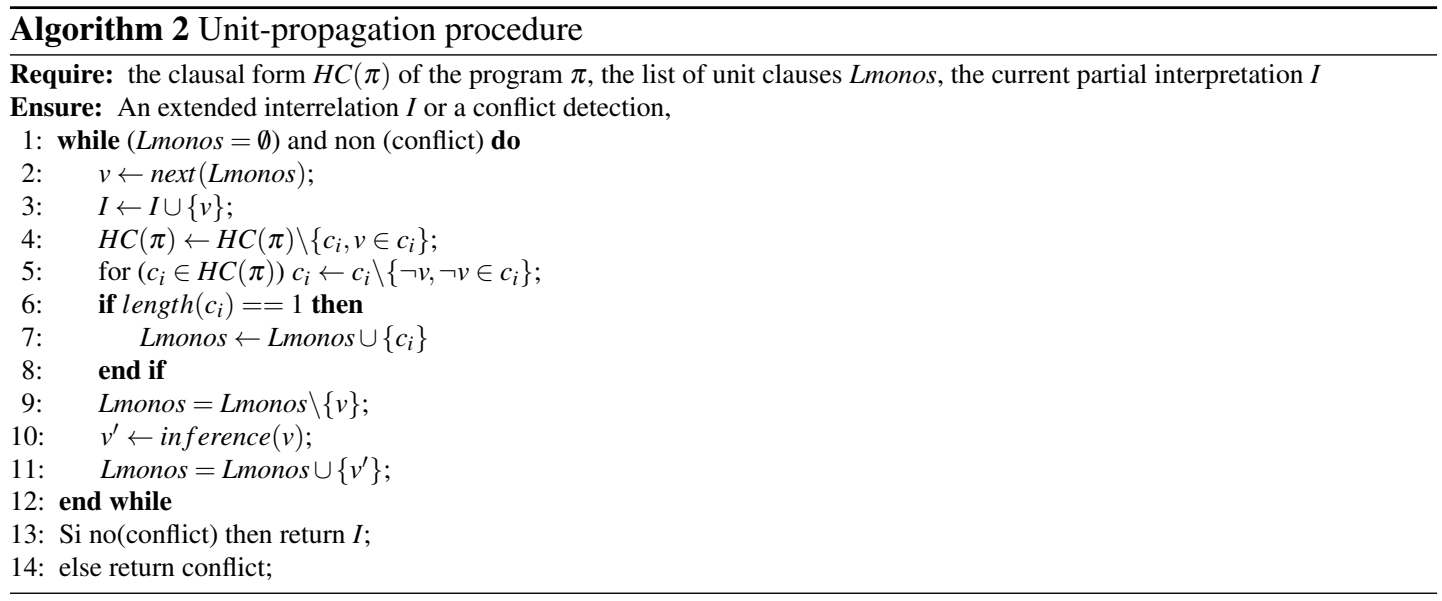

\subsubsection{The algorithm complexity}

If $n$ is the number of variables of the clausal form $H C(\pi)$ of the program $\pi, k$ the cardinality of the set $S T B$ and $m$ the number of clauses or $H C(\pi)$, then the algorithm time complexity in the worst case is approximately $\mathrm{O}\left(\mathrm{knm} 2^{k}\right)$. We can notice that the exponential factor of the complexity function depends on the number $k$ representing the size of the strong backdoor set and does not depend on the number of variables $n$ as in the other ASP solvers. The value of $k$ is generally smaller than that one of $n$, hence a better time complexity.

Unlike the majority of ASP solvers using the Clark completion with the loop management and which have an exponential spatial complexity in the worst case, our method works with constant space. Indeed, the method uses as input the Horn clausal form $H C(\pi)$ whose size is identical to that one of the initial program $\pi$ and it does not vary during the executions. The spatial complexity is constant, it is of order $O(|H C(\pi)|)=O(|\pi|)$ in the worst case. 


\section{Symmetry definition and properties}

The notion of symmetry is widely studied in the field of constraint programming. In this work, we use the clausal encoding $H C(\pi)$ that we augment by the symmetry-breaking predicates that are used to avoid enumerating the symmetrical models or no-goods (interpretations that are not models) of the resulting CNF encoding. We will give in the following the main definitions and properties of the notion of symmetry of a logic program $\pi$ expressed in its Horn clausal form $H C(\pi)$. First, we define the semantics symmetry:

Definition 4. Let $H C(\pi)$ be the Horn clausal representation of $\pi$ and $L_{H C(\pi)}$ its set of literals. A semantics symmetry $\sigma$ of a $H C(\pi)$ is a permutation defined on the set $L_{H C(\pi)}$, such that $H C(\pi)$ and $\sigma(H C(\pi))$ have the same extensions (the same stable models).

In other words, a semantics symmetry of the Horn clausal representation of a logic program is a permutation of its literals which preserves the stable models. Now we define the syntactical symmetry:

Definition 5. Let $H C(\pi)$ be the Horn clausal representation of $\pi$ and $L_{H C(\pi)}$ its set of literals. A syntactical symmetry $\sigma$ of a $H C(\pi)$ is a permutation defined on $L_{H C(\pi)}$, such that $H C(\pi)=\sigma(H C(\pi))$.

A syntactic symmetry of the Horn clausal representation of a logic program is permutation of its literals which leave all the clauses of the program unchanged.

Example 2. Consider the logic program $\pi$ of Example 1 where $L_{H C(\pi)}=\{a, b$, not $a$, not $b\}$. The permutation $\sigma=(a, b)($ nota, notb $)$ defined on $L_{H C(\pi)}$ is a syntactic symmetry of $H C(\pi)$ since $\sigma(H C(\pi))=$ $H C(\pi)$.

Definition 6. Two literals $l$ and $l^{\prime}$ of $L_{H C(\pi)}$ are symmetrical if there exists a symmetry $\sigma$ of $H C(\pi)$ such that $\sigma(l)=l^{\prime}$.

Now we define the orbit of a literal:

Definition 7. Let $H C(\pi)$ be the Horn clausal representation of $\pi$, the orbit of a literal $l \in L_{H C(\pi)}$ on which the symmetry group $(\operatorname{Sym}(H C(\pi)), 0)$ acts is $l^{\operatorname{Sym}(H C(\pi))}=\{\sigma(l): \sigma \in \operatorname{Sym}(H C(\pi))\}$

We give below a property that lies between syntactical and semantics symmetry:

Proposition 6. Each syntactical symmetry of the Horn clausal representation $H C(\pi)$ is a semantics symmetry of $H C(\pi)$.

Proof. It is trivial to see that a syntactic symmetry of $H C(\pi)$ is always a semantics symmetry of $H C(\pi)$. Indeed, if $\sigma$ is a syntactic symmetry of $H C(\pi)$, then $\sigma(H C(\pi))=H C(\pi)$, thus it results that $H C(\pi)$ and $\sigma(H C(\pi))$ have the same stable models

If $E$ is an extension of $(H C(\pi), S T B)$ and $\sigma$ a syntactic symmetry, we can get another extension of $(H C(\pi), S T B)$ by applying $\sigma$ on the literals which appear in $E$. Formally:

Proposition 7. Let $\sigma$ be a syntactical symmetry of $H C(\pi), E$ is an extension of $(H C(\pi), S T B)$ iff $\sigma(E)$ is an extension of $(H C(\pi), S T B)$.

Proof. Suppose that $E=H C(\pi) \cup S^{\prime}$ is an extension of $(H C(\pi), S T B)$. It follows that $\sigma(\mathrm{HC}(\pi)) \cup \sigma\left(\mathrm{S}^{\prime}\right)$ is an extension of $(\sigma(H C(\pi)), \sigma(S T B))$. We can then deduce that $\mathrm{HC}(\pi) \cup \sigma\left(\mathrm{S}^{\prime}\right)$ is an extension of $(H C(\pi), S T B)$ since $H C(\pi)$ and $S T B$ are invariant under $\sigma$. The converse can be shown by considering the converse permutation of $\sigma$

Each stable model of a logic program $\pi$ corresponds to an extension $E$ of $(H C(\pi), S T B)$ that satisfies the discriminant condition $\left(\forall A_{i} \in V, E \models \neg n o t A_{i} \Rightarrow E \mid=A_{i}\right)$. There is a one to one bijection between the stable models of $\pi$ and these extensions. 
Corollary 1. Let $\sigma$ be a syntactical symmetry of $H C(\pi)$, I is a stable model of $H C(\pi)$ iff $\sigma(I)$ is a stable model of $H C(\pi)$.

Example 3. In Example 2, the orbit of the literal $a$ is $a^{S y m(L)=\{a, b\}}$ and the one of the literal not $a$ is not $a^{\text {Sym }(L)}=\{$ not a,not $b\}$. All the literals of a same orbit are all symmetrical. For instance, in Example 1 there are two symmetrical extensions of $(H C(\pi), S T B)$. The fist one is $E_{1}=H C(\pi) \cup\{$ not a $\}$ and the second is $\sigma\left(E_{1}\right)=H C(\pi) \cup\{$ not $b\}$. These are symmetrical extensions of $(H C(\pi), S T B)$. We can deduce from these two extensions that there are two symmetrical stable models of $H C(\pi)$. The first one is $M_{1}=\{b\}$ and the second one is $\sigma\left(M_{1}\right)=\{a\}$.

If $(\operatorname{Perm}(H C(\pi)), \circ)$ denotes the group of permutations of $L_{H C(\pi)}$ and $\operatorname{Sym}\left(L_{H C(\pi)}\right) \subset \operatorname{Perm}\left(L_{H C(\pi)}\right)$ the subset of permutations of $L_{H C(\pi)}$ that are the syntactic symmetries of $H C(\pi)$, then $(\operatorname{Sym}(H C(\pi)), \circ)$ is a sub-group of $(\operatorname{Perm}(H C(\pi)), \circ)$ forming the symmetry group of $H C(\pi)$.

\section{Symmetry detection}

Our symmetry detection is based on graph automorphism search. We first represent the Horn clausal form $H C(\pi)$ by a colored graph $G_{H C(\pi)}$ then compute its set of automorphisms which should be identical to the symmetry group of $H C(\pi)$. This technique has been widely studied in the context of constraint satisfaction and satisfiability problems $[9,2]$. Let $G_{H C(\pi)}(V, E)$ be a graph associated to $H C(\pi)$, where $V$ is a set of vertices and $E \subseteq V \times V$ a set of edges. An automorphism (symmetry) of $G_{H C(\pi)}$ is a permutation of the vertices that leaves the graph unchanged. Only the vertices having the same color are permuted together. Given the Horn clausal representation $H C(\pi)$ of the program $\pi$, the associated colored graph $G_{H C(\pi)}(V, E)$ of $H C(\pi)$ is defined as follows:

- Each positive literal $A_{i}$ of $H C(\pi)$ is represented by a vertex $A_{i} \in V$ of the color 1 in $G_{H C(\pi)}$. The negative literal $\neg A_{i}$ associated with $A_{i}$ is also represented by a vertex $\neg A_{i}$ of color 2 in $G_{H C(\pi)}$. These two vertices are connected by an edge of $E$ in the graph $G_{H C(\pi)}$.

- Each literal $\neg$ not $A_{i} \in S T B$ associated with $A_{i}$ is represented by a vertex $\neg$ not $A_{i}$ of color 3 in $G_{H C(\pi)}$. This vertex is connected to that one representing $A_{i}$ by an edge of $E$ in the graph $G_{H C(\pi)}$.

- Each literal $\neg$ not $A_{i} \notin S T B$ associated with $A_{i}$ is represented by a vertex $\neg$ not $A_{i}$ of color 4 in $G_{H C(\pi)}$. This vertex is connected to that one representing $A_{i}$ by an edge of $E$ in the graph $G_{H C(\pi)}$.

- Each clause rule $c_{i} \in C R$ of $H C(\pi)$ is represented by a vertex $c_{i} \in C R$ of color 5 in $G_{H C(\pi)}$. An edge connects this vertex $c_{i}$ to each vertex representing one of its literals.

- Each mutual clause $c_{i} \in M E$ of $H C(\pi)$ is represented by a vertex $c_{i} \in V$ of color 6 in $G_{H C(\pi)}$. An edge connects this vertex $c_{i}$ to the two vertices representing its literals.

This graph construction ensures that only vertices having the same color could be permuted together. The graph $G_{H C(\pi)}$ preserves the syntactic group of symmetries of $H C(\pi)$. That is, the syntactic symmetry group of the representation $H C(\pi)$ of a logic program $\pi$ is identical to the automorphism group of its graph representation $G_{H C(\pi)}$. The edge between $A_{i}$ and $\neg A_{i}$ guarantee that an automorphism that maps $A_{1}$ to $A_{2}$ also maps $\neg A_{1}$ to $\neg A_{2}$. We could use then a graph automorphism system like saucy, autom or nauty to detect the syntactic symmetry group of $H C(\pi)$. These systems return a set of generators of the symmetry group from which we can deduce each symmetry of $H C(\pi)$.

Example 4. Consider the logic program $\pi$ of Example 1. The Horn clausal representation of the logic program $\pi$ is formed by the set $H C(\pi)=C R \cup M E$ where $C R=\{1: a \vee \neg$ not $b, 2: b \vee \neg$ not $a\}$, 


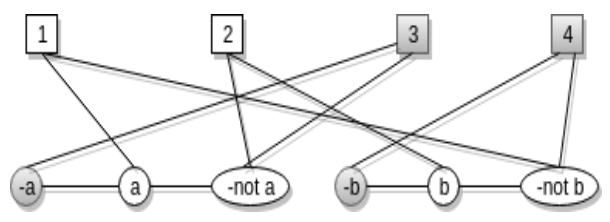

Figure 1: The graph representation of $H C(\pi)$

$M E=\{3: \neg a \vee \neg$ not $a, 4: \neg b \vee \neg$ not $b\}$ and its strong backdoor is given by STB $=\{$ not a, not $b\}$. Its corresponding graph $G_{H C(\pi)}$ is given in Figure 1. We used in this example five colors that are represented by circles, shaded circles, ellipses, square, and shaded square. The vertices 1,2,3 and 4 represents the fourth clauses of $H C(\pi)$. We can see for instance that the vertex permutation $\sigma=$ $(a, b)(\neg a, \neg b)(\neg$ nota,$\neg$ notb $)(1,2)(3,4)$ is an automorphism of $G_{H C(\pi)}$. The restriction of the automorphism $\sigma$ to the elements of the STB represents the symmetry $\sigma=(\neg$ nota, $\neg$ notb $)$

\section{Symmetry breaking}

The different approaches proposed to break symmetries can be classified in two categories: dynamic and static symmetry breaking. The dynamic approach usually looks after and breaks symmetries at each node of the search tree, while the static approach detects and breaks the symmetries in a pretreatment step. In the static approach, symmetries are generally broken by generating additional constraints, called symmetry-breaking predicates (SBP). Here we deal with the static symmetry-breaking technique. The construction of the symmetry-breaking predicates is based on the lex-leader method introduced by Crawford et al. in [9] and improved by Aloul et al. [1].

Given a logic program $\pi$, the symmetries of its Horn clausal form $H C(\pi)$ induce equivalence classes in the solution/no-good spaces of the program. All the symmetrical interpretations of a stable model/nogood $I$ of $\pi$ are stable models/no-good of $\pi$. Symmetry is then an equivalence relation that defines a partition on the set of interpretations. It is then possible to represent each equivalent class by a representative interpretation. That is, an interpretation $I_{1}$ is equivalent to another interpretation $I_{2}$ if there exists a symmetry $\sigma$ of $\operatorname{Sym}(H C(\pi))$ such that $I_{2}=\sigma\left(I_{1}\right)$. The symmetry-breaking predicates are chosen such that they are true for exactly one interpretation in each equivalent class (the least interpretation in the lex ordering).

Here, we focus only on the STB literals, as they represents the most difficult part in the solutions search. It is sufficient to break the symmetries existing between the STB literals. To do that, we consider an ordering on the literals not $A_{i}$ of the STB and use it to construct a lexicographical order on the set of interpretations. Consider, the symmetry group $\operatorname{Sym}(H C(\pi))=\left\{\sigma_{1}, \sigma_{2}, \ldots, \sigma_{n}\right\}$ of $H C(\pi)$ and a total ordering not $A_{1} \leq$ not $A_{2} \leq \ldots \leq$ not $A_{n}$ on the literals not $A_{i}$ of the STB. The advantage here results in the fact that the size of the STB set is generally smaller than that one of all the literals of $H C(\pi)$. This will lead to get a symmetry elimination predicate of a small size. We obtain the partial lex-leader symmetrybreaking predicates $P L L-S B P$ by encoding a permutation constraint $P C(\sigma)$ for every permutation $\sigma$, defined by:

$$
P C(\sigma)=\bigwedge_{1 \leq i \leq n}\left[\bigwedge_{1 \leq j \leq i-1}\left(\operatorname{not} A_{j}=\operatorname{not} A_{j}^{\sigma}\right)\right] \rightarrow\left(\operatorname{not} A_{i} \leq \operatorname{not} A_{i}^{\sigma}\right)
$$

The PLL-SBP of $H C(\pi)$ is then represented by the conjunction of the permutation constraints asso- 
ciated to the considered permutations. It is expressed as follows:

$$
P L L-S B P=\bigwedge_{\sigma \in G e n(\operatorname{Sym}(H C(\pi))} P C(\sigma)
$$

Each $P C(\sigma)$ is translated to a CNF formula whose size is linear in the size $n$ of the STB. The first step is done by introducing the two ordering predicates $l_{i}=\left(\operatorname{not} A_{i} \leq\right.$ not $\left.A_{i}^{\sigma}\right)$ and $g_{i}=\left(\operatorname{not} A_{i} \geq \operatorname{not} A_{i}^{\sigma}\right)$. The purpose of adding these two predicates is the elimination of the "equality" and the "less than or equal" operators in predicate $P C(\sigma)$. We obtain the following formula:

$$
P C(\sigma)=\bigwedge_{1 \leq i \leq n}\left[\bigwedge_{1 \leq j \leq i-1} l_{j} g_{j}\right] \rightarrow l_{i}
$$

More precisely :

$$
P C(\sigma)=\left(1 \rightarrow l_{1}\right)\left(l_{1} g_{1} \rightarrow l_{2}\right)\left(l_{1} g_{1} l_{2} g_{2} \rightarrow l_{3}\right) \ldots\left(l_{1} g_{1} l_{2} g_{2} \ldots l_{n-1} g_{n-1} \rightarrow l_{n}\right)
$$

In the next step, we will use the following known lemma :

Lemma 1. $(a \rightarrow b) \wedge \bigwedge_{i \in I}\left(a b c_{i} \rightarrow d_{i}\right)=(a \rightarrow b) \wedge \bigwedge_{i \in I}\left(a c_{i} \rightarrow d_{i}\right)^{3}$

Repeated applications of Lemma 1 to the formula 4 leads to a successive elimination of the predicates $l_{j}$ in the left side of the implications of the formula. We obtain the following:

$$
\begin{aligned}
& P C(\sigma) \\
& =\left(1 \rightarrow l_{1}\right)\left(g_{1} \rightarrow l_{2}\right)\left(g_{1} l_{2} g_{2} \rightarrow l_{3}\right) \ldots\left(g_{1} l_{2} g_{2} \ldots l_{n-1} g_{n-1} \rightarrow l_{n}\right) \\
& =\left(1 \rightarrow l_{1}\right)\left(g_{1} \rightarrow l_{2}\right)\left(g_{1} g_{2} \rightarrow l_{3}\right) \ldots\left(g_{1} g_{2} \ldots l_{n-1} g_{n-1} \rightarrow l_{n}\right) \\
& =\cdots \\
& =\left(1 \rightarrow l_{1}\right)\left(g_{1} \rightarrow l_{2}\right)\left(g_{1} g_{2} \rightarrow l_{3}\right) \ldots\left(g_{1} g_{2} \ldots g_{n-1} \rightarrow l_{n}\right) \\
& =\bigwedge_{1 \leq i \leq n}\left[\bigwedge_{1 \leq j \leq i-1} g_{j}\right] \rightarrow l_{i}
\end{aligned}
$$

Next, we introduce $n$ auxiliary chaining variables $p_{i}$ that are defined by:

$$
\begin{aligned}
& p_{0}=1 \\
& p_{1}=p_{0} \wedge g_{1}=g_{1} \\
& p_{2}=p_{1} \wedge g_{2}=g_{1} \wedge g_{2} \\
& \cdots \\
& p_{n-1}=p_{n-2} \wedge g_{n-1}=\bigwedge_{1 \leq j \leq n-1} g_{j}
\end{aligned}
$$

The substitution of the order predicates by these definitions gives the following expression:

$$
P C(\sigma)=\left[\bigwedge_{1 \leq i \leq n}\left(p_{i-1} \rightarrow\left(\operatorname{not} A_{i} \leq n o t A_{i}^{\sigma}\right)\right)\right] \wedge\left[\bigwedge_{1 \leq i \leq n-1}\left(p_{i-1} \wedge\left(n o t A_{i} \geq n o t A_{i}^{\sigma}\right)=p_{i}\right)\right]
$$

\footnotetext{
${ }^{3}$ The expression $a b c_{i}$ and $a c_{i}$ means respectively $a \wedge b \wedge c_{i}$ and $a \wedge c_{i}$
} 
which can be converted to a CNF formula consisting of $4 n$ 3-literal clauses and $n$ 2-literal clauses for a total of $14 n$ literals. A further reduction is possible by replacing the equality predicate of the formula 5 by a one-way implication, which gives rise to the following formula:

$$
P C(\sigma)=\left[\bigwedge_{1 \leq i \leq n}\left(p_{i-1} \rightarrow\left(\operatorname{not} A_{i} \leq \operatorname{not} A_{i}^{\sigma}\right)\right)\right] \wedge\left[\bigwedge_{1 \leq i \leq n-1}\left(p_{i-1} \wedge\left(\operatorname{not} A_{i} \geq \operatorname{not} A_{i}^{\sigma}\right) \rightarrow p_{i}\right)\right]
$$

from which, we can obtain a CNF formula that consist of $3 n$ 3-literal clauses for a total of $9 n$ literals.

\section{Experimentation}

Based on the algorithm presented previously, we implemented a new ASP solver that we denote by $H C$-asp to mean Horn Clause ASP. We also included in our system the symmetry breaking to obtain the variant $\mathrm{HC}$-asp-sym, the entire system is implemented in $\mathrm{C}++$. The symmetry-breaking method used here, is a static approach that eliminates symmetries in a preprocessing phase. Our ASP system takes as input a ground logic program $\pi$ produced by the grounder gringo [15]. It uses the clausal representation $H C(\pi)$ to compute the stable models of $\pi$. The system builds the colored graph $G_{H C(\pi)}$ of $H C(\pi)$ that saucy [10] uses to detect the symmetry group of $H C(\pi)$. The symmetry-breaking predicates are computed according to the detected group generators then added to the encoding $H C(\pi)$. After this, an ASP solver could be used on the resulting encoding as a black-box. The general schema of the process is illustrated in Figure 2.

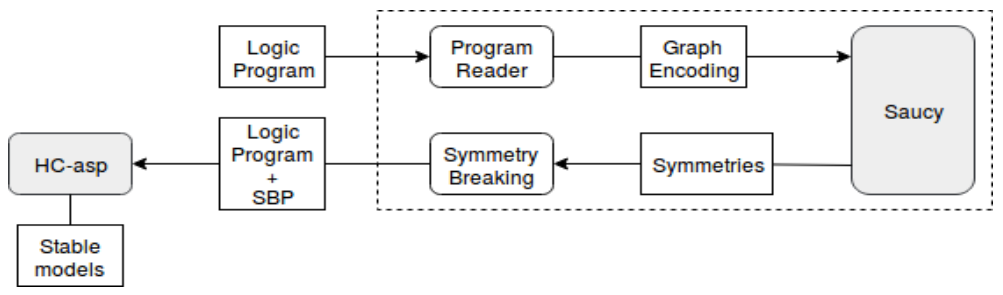

Figure 2: The general schema of ASP solver with the advantage of symmetry

To evaluate our approach, we experimented HC-asp-sym on combinatorial problems then compared it performances to that one of HC-asp and the ones of Cmodels (version 3.86 with zChaff as a SAT solver), Smodels (version 2.34) and Clasp(version 3.3.3). The system HC-asp-sym is also compared to the symmetry-breaking system Sbass associated to Clasp. The system Sbass is presented in [12]. This Sbass system is based on a static approach that eliminates symmetries in a preprocessing phase. The symmetry-breaking predicates are computed and added to the logic program $\pi$. Clasp is then applied to the resulting program. The systems are applied to search all the stable models and gringo is used as a grounder for all the systems. The programs run on a 4GB Ubuntu (16.10) machine with an Intel Core i5 $(1.70 \mathrm{GHz} x 4)$. The CPU run time is limited to 24 hours for all the applied systems. The symbol • in the tables means that the corresponding solver fails to solve the instance by the time limit. We report here the results obtained on some known benchmarks that are the Reachability problem, the consistent Pigeon Hole problem, the Ramsey problem, the n-queen problem and the Hamiltonian circuit. The most important task in answer set programming is to enumerate all the stable models of a logic program. symmetry-breaking techniques are also more relevant for calculating all the solutions of a problem. The impact of the symmetry breaking should be very interesting because a solver never explores two points in the search tree that are symmetrical. We chose these benchmarks because of the large number of 
stable models. They are very appropriate to study the behavior of each of the solvers when the number of stable models and the size of the problem increase. We used the same problem encoding for all the solvers.

Table 1: The results obtained on the Reachability, Ramsey, and Pigeon Hole

\begin{tabular}{|c|c|c|c|c|c|c|c|c|c|c|c|c|c|c|}
\hline \multirow[t]{2}{*}{$N^{\circ}$} & \multirow[t]{2}{*}{ Instances } & \multirow[t]{2}{*}{ \#Stable Models } & \multirow[b]{2}{*}{ HC-asp } & \multirow[b]{2}{*}{ Clasp } & \multirow[b]{2}{*}{ Smodels } & \multirow[b]{2}{*}{ Cmodels } & \multicolumn{4}{|c|}{ HC-asp-sym } & \multicolumn{4}{|c|}{ Sbass \& Clasp } \\
\hline & & & & & & & \#Sym & \#SBP & \#nssmodels & Time & \#Sym & \#SBP & \#nssmodels & Time \\
\hline 1 & R_2 & 1 & 0.0005 & 0.0001 & 0.0002 & 0.0003 & 1 & 6 & 1 & 0.0002 & 1 & 8 & 1 & 0.0001 \\
\hline 2 & R_3 & 18 & 0.0015 & 0.001 & 0.0005 & 0.015 & 2 & 23 & 9 & 0.0004 & 2 & 76 & 8 & 0.002 \\
\hline 3 & R_4 & 1606 & 0.030 & 0.070 & 0.022 & 0.091 & 2 & 54 & 725 & 0.011 & 2 & 148 & 724 & 0.010 \\
\hline 4 & R_5 & 565080 & 7.12 & 12.59 & 7.62 & 9991.28 & 3 & 134 & 176733 & 2.94 & 3 & 276 & 120446 & 3.6 \\
\hline 5 & R_4_5_5 & 957 & 0.011 & 0.010 & 0.014 & 0.049 & 3 & 33 & 66 & 0.001 & 4 & 88 & 188 & 0.0001 \\
\hline 6 & R_4_5_6 & 27454 & 0.2 & 0.5 & 0.33 & 18.62 & 5 & 72 & 1023 & 0.12 & 5 & 156 & 1476 & 0.04 \\
\hline 7 & R_4_5_7 & 1452289 & 12.64 & 28.76 & 18.00 & - & 5 & 99 & 99337 & 1.52 & 6 & 312 & 25192 & 0.61 \\
\hline 8 & R_4_5_8 & 137578233 & 1625.14 & 3329.66 & 2219.19 & - & 6 & 155 & 3071804 & 54.67 & 6 & 360 & 12230197 & 332.51 \\
\hline 9 & pi $4 / 4$ & 24 & 0.007 & 0.009 & 0.002 & 0.003 & 4 & 84 & 6 & 0.0005 & 6 & 96 & 4 & 0.0001 \\
\hline 10 & $\mathrm{pi} 5 / 5$ & 120 & 0.02 & 0.02 & 0.008 & 0.01 & 5 & 147 & 12 & 0.0011 & 8 & 160 & 8 & 0.001 \\
\hline 11 & pi6/6 & 720 & 0.06 & 0.06 & 0.04 & 0.07 & 6 & 189 & 70 & 0.0048 & 10 & 240 & 54 & 0.002 \\
\hline 12 & pi7/7 & 5040 & 0.23 & 0.55 & 0.30 & 0.87 & 7 & 249 & 840 & 0.069 & 11 & 336 & 288 & 0.036 \\
\hline 13 & pi $8 / 8$ & 40320 & 1.65 & 4.01 & 2.5 & 52.34 & 8 & 336 & 2424 & 0.42 & 12 & 448 & 1800 & 0.080 \\
\hline 14 & pi $9 / 9$ & 362880 & 21.63 & 47.11 & 34.60 & 4591.87 & 9 & 702 & 19634 & 1.26 & 13 & 832 & 12960 & 0.51 \\
\hline 15 & pi10/10 & 3628800 & 210.14 & 494.40 & 369.80 & • & 10 & 846 & 170354 & 19.92 & 14 & 1008 & 105840 & 16.3 \\
\hline 16 & pi11/11 & 39916800 & 2728.53 & 6936.96 & 4247.58 & - & 11 & 1044 & 976680 & 55.77 & 15 & 1360 & 893760 & 76.25 \\
\hline
\end{tabular}

Tables 1, 2, 3 and 4 give the runtime of the different benchmarks for all the ASP solvers and the number of stable models found by all of them if they finish before the time limit. They also show some symmetry information of the system HC-asp-sym and Sbass \& Clasp: \#sym represents the number of detected symmetry, \#SBP the number of added SBPs and \#nssmodels the number of non symmetrical stable models. The run time is the one of the enumeration of all the solutions including the symmetry preprocessing. The results obtained on the four benchmarks, Reachability(1-4), Pigeon hole(9-16), Ramsey(4-8) are given in Table 1. In general, $H C$ - asp outperforms all the other ASP systems. We can observe that the use of symmetry breaking reduces considerably the solution space for all the benchmarks, and therefore reduces the CPU runtime. This applies to the combination sbass and clasp and also to our system including the elimination of symmetries. That is, HC-asp-sym has better results than those of $H C$-asp and the ones of the other systems. Clearly, we can see that symmetry breaking reduces significantly the number of computed stable models. The gain increases when the problem size and the number of stable models increase.

The Pigeon Hole problem is a good illustration for that observation. For Pigeon Hole of sizes 9 to 11, the solution space is compressed considerably. This reduces the CPU runtime. For the Reachability, and Ramsey problem, we can see that HC-asp, Clasp, and Smodels have comparable results. Again, HC-asp-sym get better results on these problems. The comparison of the system Sbass associated to clasp with $\mathrm{HC}$-asp-sym shows that for the problem of Reachability and the Pigeon Hole, both systems have comparable results in terms of CPU time. But for the Ramsey problem, HC-asp-sym has better results than Sbass \& Clasp.

Table 2: The results obtained on the n-queens problem

\begin{tabular}{|c|c|c|c|c|c|c|c|c|c|c|c|c|c|}
\hline \multirow[t]{2}{*}{ \#Size } & \multirow[t]{2}{*}{ \#Stable Models } & \multirow[b]{2}{*}{ HC-asp } & \multirow[b]{2}{*}{ Clasp } & \multirow[b]{2}{*}{ Smodels } & \multirow[b]{2}{*}{ Cmodels } & \multicolumn{4}{|c|}{ HC-asp-sym } & \multicolumn{4}{|c|}{ Sbass \& Clasp } \\
\hline & & & & & & \#Sym & \#SBP & \#sol & Time & \#Sym & \#SBP & \#sol & Time \\
\hline 10 & 724 & 0.68 & 0.23 & 0.66 & 0.27 & 3 & 468 & 240 & 0.10 & 2 & 812 & 278 & 0.08 \\
\hline 11 & 2680 & 2.79 & 1.02 & 2.95 & 2.48 & 3 & 528 & 852 & 0.56 & 2 & 936 & 1032 & 0.35 \\
\hline 12 & 14200 & 12.2 & 8.75 & 15.19 & 41.44 & 3 & 810 & 4981 & 3.69 & 2 & 1168 & 5452 & 2.7 \\
\hline 13 & 73712 & 79.55 & 122.91 & 87.69 & 1642.93 & 3 & 921 & 24934 & 20.53 & 2 & 1316 & 28259 & 31.14 \\
\hline 14 & 365596 & 371.73 & 2631.83 & 496.98 & - & 3 & 918 & 107371 & 120.26 & 2 & 1588 & 125728 & 613.51 \\
\hline 15 & 2279184 & 2797.02 & 34337.21 & 3352.37 & • & 3 & 1221 & 788141 & 854.31 & 2 & 1760 & 871604 & 13007.53 \\
\hline 16 & 14772512 & 12087.40 & $\bullet$ & 23134.22 & $\bullet$ & 3 & 1188 & 4310853 & 4976.44 & 2 & 2072 & - & $\bullet$ \\
\hline 17 & 95815104 & 87088.00 & $\bullet$ & $\bullet$ & $\bullet$ & 3 & 1275 & 29227320 & 29429.41 & 2 & 2268 & $\bullet$ & $\bullet$ \\
\hline
\end{tabular}

We also experimented the n-queens and the super n-queens problems. The n-queens problem con- 
sists in placing $n$ queens on an $n \times n$ chessboard so that no two queens threaten each other. The super $\mathrm{n}$-queens is a variant of the $\mathrm{n}$-queens where a super queen simultaneously plays the role of a queen and a knight. The obtained results are shown in Table 2 and 3.

Table 3: The results obtained on the super n-queens problem

\begin{tabular}{|c|c|c|c|c|c|c|c|c|c|c|c|c|c|}
\hline \multirow{2}{*}{ \#Size } & \multirow{2}{*}{ \#Stable Models } & \multirow[b]{2}{*}{ HC-asp } & \multirow[b]{2}{*}{ Clasp } & \multirow[b]{2}{*}{ Smodels } & \multirow[b]{2}{*}{ Cmodels } & \multicolumn{4}{|c|}{ HC-asp-sym } & \multicolumn{4}{|c|}{ Sbass \& Clasp } \\
\hline & & & & & & \#Sym & \#SBP & \#sol & Time & \#Sym & \#SBP & \#sol & Time \\
\hline 10 & 4 & 0.32 & 0.01 & 0.12 & 0.023 & 3 & 467 & 2 & 0.013 & 2 & 812 & 2 & 0.01 \\
\hline 11 & 44 & 0.54 & 0.03 & 0.36 & 0.049 & 3 & 554 & 13 & 0.046 & 2 & 936 & 14 & 0.03 \\
\hline 12 & 156 & 1.12 & 0.09 & 1.65 & 0.17 & 3 & 675 & 49 & 0.25 & 2 & 1168 & 63 & 0.11 \\
\hline 13 & 1876 & 3.61 & 0.54 & 8.12 & 1.67 & 3 & 898 & 651 & 1.57 & 2 & 1316 & 651 & 0.56 \\
\hline 14 & 5180 & 16.84 & 5.94 & 41.53 & 21.13 & 3 & 1086 & 1960 & 7.61 & 2 & 1588 & 1767 & 2.84 \\
\hline 15 & 32516 & 98.47 & 122.42 & 231.52 & 1241.05 & 3 & 1124 & 11217 & 38.24 & 2 & 1760 & 11271 & 39.46 \\
\hline 16 & 202900 & 653.56 & 1571.07 & 23134.22 & 44433.07 & 3 & 1176 & 62220 & 207.97 & 2 & 2072 & 77287 & 654.22 \\
\hline 17 & 1330622 & 4455.07 & 48005.13 & 10001.26 & $\bullet$ & 3 & 1435 & 456661 & 2313.38 & 2 & 2268 & 503462 & 18403.85 \\
\hline 18 & 8924976 & 32649.14 & - & 77747.20 & • & 3 & 1503 & 2596466 & 13792.58 & 2 & 2620 & $\bullet$ & $\bullet$ \\
\hline
\end{tabular}

We can see in Table 2 that all the methods solved efficiently the small instances of the the n-queens problem (10 to 12 queens) and those of the super n-queens problem (10 to 14 super queens). The results of HC-asp, Clasp and Smodels are comparable on these instances with a slight advantage in the favor of the system Clasp. We can observe for both benchmarks that HC-asp outperforms drastically all the other methods on the big instances. We can remark that the gain realized by HP-asp increases when the size of the problem increases and when the number of solutions increases also. Symmetry breaking greatly contributed to improve the results of $H C$-asp. Again, the number of computed solutions and the time spent on the exploration of all the search space are reduced when applying HC-asp-sym. We can see in Table 2 that sbass associated with clasp has better result than our system on small instances. But from the instance 13, HC-asp-sym is more efficient. It can be seen that only the solver HC-asp-sym is able to solve all the problems by the time limit. Indeed, Sbass associated to clasp timed out for the two instances corresponding to 16 and 17 queens. Table 3, shows the results obtained on the super n-queens problem. They look very similar to that ones of the n-queens problem. HC-asp-sym is able to solve all the problems by the time limit, Sbass \& clasp timed out for the instance having 17 super queens. The number of SBPs added in the case of Sbass is greater than that one corresponding to the SBPs added by our method. The most plausible hypothesis to explain this observation, is that saucy is sensitive to the differences existing in the graph encodings of both approaches. The second fact could be the lex-leader of our method that is restricted to the only the STB set. The last benchmark is the Hamiltonian circuit [22]. We computed all the Hamiltonian circuits of some complete oriented graphs whose number of vertices varies from 5 to 12 . The behavior of all the solvers are represented in Table 4 .

Table 4: The results obtained on the Hamiltonian problem

\begin{tabular}{|c|c|c|c|c|c|c|c|c|c|c|c|c|c|}
\hline \multirow[t]{2}{*}{ \#Size } & \multirow[t]{2}{*}{ \#Stable Models } & \multirow[b]{2}{*}{ HC-asp } & \multirow[b]{2}{*}{ Clasp } & \multirow[b]{2}{*}{ Smodels } & \multirow[b]{2}{*}{ Cmodels } & \multicolumn{4}{|c|}{ HC-asp-sym } & \multicolumn{4}{|c|}{ Sbass \& Clasp } \\
\hline & & & & & & \#Sym & \#SBP & \#sol & Time & \#Sym & \#SBP & \#sol & Time \\
\hline 5 & 24 & 0.012 & 0.003 & 0.003 & 0.003 & 3 & 90 & 6 & 0.0005 & 3 & 108 & 4 & 0.00001 \\
\hline 6 & 120 & 0.026 & 0.016 & 0.014 & 0.02 & 3 & 132 & 48 & 0.0031 & 4 & 224 & 48 & 0.0001 \\
\hline 7 & 720 & 0.09 & 0.10 & 0.08 & 0.12 & 4 & 186 & 288 & 0.014 & 3 & 280 & 240 & 0.010 \\
\hline 8 & 5040 & 0.64 & 0.78 & 0.67 & 1.69 & 4 & 246 & 1992 & 0.13 & 6 & 336 & 1440 & 0.11 \\
\hline 9 & 40320 & 5.76 & 7.87 & 6.00 & 80.75 & 5 & 348 & 5040 & 0.64 & 7 & 392 & 7920 & 0.8 \\
\hline 10 & 362880 & 66.67 & 89.94 & 72.25 & 6177.89 & 5 & 396 & 40320 & 6.63 & 8 & 448 & 57600 & 10.91 \\
\hline 11 & 3628800 & 910 & 1141.84 & 964.15 & - & 6 & 591 & 412118 & 107.26 & 9 & 504 & 786240 & 123.92 \\
\hline 12 & 39916800 & 13173.42 & 22263.37 & 15964.15 & - & 6 & 612 & 7327392 & 4604.26 & 10 & 560 & 7781760 & 4842.33 \\
\hline
\end{tabular}

The results show that all the methods solved efficiently the instances having a number of vertices less than eight (small instances) and HC-asp is competitive on the big instances. Again HC-asp-sym gets better results than the other methods. The advantage of symmetry is more important when the problem size increases. The comparison with sbass associated to clasp shows that both systems have comparable results with a slight advantage for our system in terms of CPU time. 


\section{Conclusion}

In this paper, we provided a new method to compute stable models. This method is based on a relatively new semantics and has the advantage of using a Horn clausal logic form whose size is identical to that one of the source ground logic program. It also has a constant spatial complexity. The semantics used prevent the method from the additional burden induced by the the loop management in the semantics of Clark completion, this semantics is used by several ASP solvers. The other benefit of our approach is the simplified enumerative process which is only done on a subset of the variables representing the strong backdoor of the source logic program. This lead to a considerable gain in the time complexity. We also proposed the integration of symmetry breaking in order to avoid exploring isomorphic subspaces. We experimented the proposed method with and without symmetry breaking on a variety of known combinatorial problems. The obtained results showed that our approach is a good alternative to implement ASP solvers and symmetry breaking leads to a significant improvement.

As a future work, we will look first to enhance our implementation with the techniques used in modern SAT solvers such as watched literals, lazy structures, clause learning and restart. This could be beneficial when searching for one stable model like in satisfiability problems. Here we limited the system to search only the stable models of a logic program in order to compare it to other ASP solvers. We are looking to extend the method to search for extra-models that could give a meaning to logical programs in the absence of stable models. Finally, we are looking to investigate some extensions of our approach to others classes of logic programming or to pieces of more general non-monotonic logics.

\section{References}

[1] F.A Aloul, , I.L. Markov, and K.A. Sakallah. Efficient symmetry-breaking for boolean satisfiability. IJCAI, pages 271-276, 2003.

[2] F.A Aloul, A. Ramani, I.L. Markov, and K.A. Sakallah. Solving difficult sat instances in the presence of symmetry. DAC, pages 731-736, 2002.

[3] F.A Aloul, A. Ramani, I.L. Markov, and K.A. Sakallah. Solving difficult sat instances in the presence of symmetry. DAC, pages 1117-1137, 2003.

[4] B. Benhamou and L. Sais. Theoretical study of symmetries in propositional calculus and application. $C A D E$, 607:281-294, 1992.

[5] B. Benhamou and L. Sais. Tractability through symmetries in propositional calculus. The Journal of Automated Reasoning, pages 89-102, 1994.

[6] Belaid Benhamou. Dynamic and static symmetry breaking in answer set programming. LPAR, pages 112-126, 2013.

[7] Belaïd Benhamou and Pierre Siegel. A new semantics for logic programs capturing and extending the stable model semantics. Tools with Artificial Intelligence (ICTAI), pages 25-32, 2012.

[8] Keith L Clark. Negation as failure. Logic and data bases, pages 293-322, 1978.

[9] James M. Crawford, Matthew L. Ginsberg, Eugene M. Luks, and Amitabha Roy. Symmetry-breaking predicates for search problems. KR, pages 148-159, 1996.

[10] P.T Darga, K.A. Sakallah, and I.L. Markov. Faster symmetry discovery using sparsity of symmetries. DAC, pages 149-154, 2008.

[11] Martin Davis, George Logemann, and Donald Loveland. A machine program for theorem proving. Communications of the ACM, 5:394-397, 1962.

[12] Christian Drescher, Oana Tifrea, and Toby Walsh. Symmetry-breaking answer set solving. AI Communications, 24:177-194, 2011.

[13] Francois Fages. Consistency of clark's completion and existence of stable models. Methods of Logic in Computer Science, 1:51-60, 1994. 
[14] Martin Gebser, Benjamin Kaufmann, Andrá Neumann, and Torsten Schaub. Conflict-driven answer set solving. IJCAI, 7:386-392, 2007.

[15] Martin. Gebser, Torsten Schaub, and Sven Thiele. Gringo: A new grounder for answer set programming. International Conference on Logic Programming and Nonmonotonic Reasoning, 7:266-271, 2007.

[16] Michael Gelfond and Vladimir Lifschitz. The stable model semantics for logic programming. ICLP/SLP, 50:1070-1080, 1988.

[17] B. Krishnamurty. Short proofs for tricky formulas. Acta Inf., 22:253-275, 1985.

[18] Nicola. Leone, Gerald Pfeifer, Wolfgang Faber, Thomas Eiter, Georg Gottlob, Simona Perri, and Francesco Scarcello. The dlv system for knowledge representation and reasoning. ACM Transactions on Computational Logic (TOCL), 7:499-562, 2006.

[19] Yuliya Lierler and Marco Maratea. Cmodels-2: Sat-based answer set solver enhanced to non-tight programs. Logic Programming and Nonmonotonic Reasoning, pages 346-350, 2004.

[20] Fangzhen Lin and Yuting Zhao. Assat: Computing answer sets of a logic program by sat solvers. Artificial Intelligence, pages 115-137, 2004.

[21] B. McKay. Practical graph isomorphism. Numerical mathematics and computing., pages 45-87, 1981.

[22] Ilkka Niemelä. Logic programs with stable model semantics as a constraint programming paradigm. Annals of Mathematics and Artificial Intelligence, 25:241-273, 1991.

[23] Ilkka Nimelä, Patrik Simons, and Tommi Syrjanen. Smodels: A system for answer set programming. Proceedings of the 8th International Workshop on Non-Monotonic Reasoning, 2000.

[24] J.F. Puget. Automatic detection of variable and value symmetries. CP, pages 475-489, 2005.

[25] Patrik Simons, Ilkka Nimelä, and Timo Soininen. Extending and implementing the stable model semantic. Artificial Intelligence, 138:181-234, 2002.

[26] Ryan Williams, Carla P Gomes, and Bart Selman. Backdoors to typical case complexity. International joint conference on artificial intelligence, 18:1173-1178, 2003. 